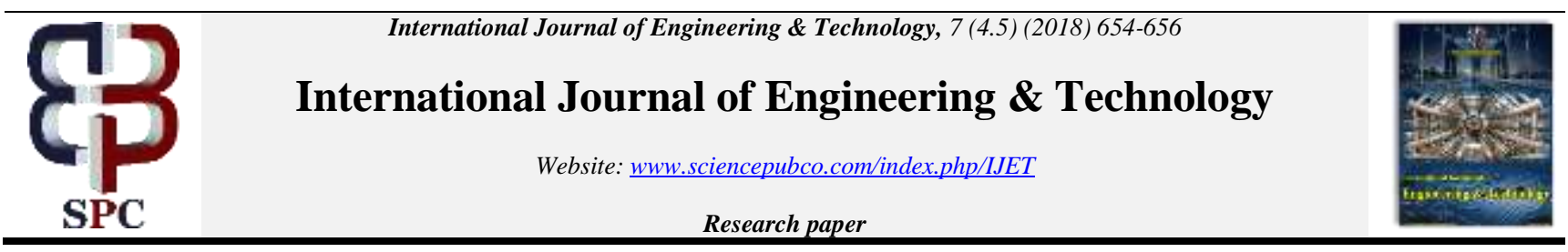

\title{
Implementation of machine learning techniques to control the speed of the vehicles
}

\author{
M. S. Satyanarayana ${ }^{1 *}$, Aruna T. $\mathbf{M}^{2}$, Divyaraj G. $\mathbf{N}^{2}$ \\ ${ }^{1}$ Research Scholar, Dept. of CS\&E, SVCE, Bengaluru \\ ${ }^{2}$ Asst. Professor, Dept. of IS\&E, SVCE, Bengaluru \\ *Corresponding author E-mail: satyanarayanams@outlook.com
}

\begin{abstract}
Accidents have become major issue in Developing countries like India now a day. As per the Surveys $60 \%$ of the accidents are happening due to over speed. Though the government has taken so many initiatives like Traffic Awareness \& Driving Awareness Week etc.., but still the percentage of accidents are not getting reduced. In this paper a new technique has been introduced to reduce the percentage of accidents. The new technique is implemented using the concept of Machine Learning [1]. The Machine Learning based systems can be implemented in all vehicles to avoid the accidents at low cost [1]. The main objective of this system is to calculate the speed of the vehicle at three various locations based on the place where the vehicle speed must be controlled and if the speed is greater than the designated speed in that road then the vehicle automatically detects the problem and same will be intimated to the driver to control the speed of the vehicle. If the speed is less or equal to the designated speed in that road then the vehicle will be passed without any disturbance. The system will be giving beep sound along with color indication to driver in each and every scenario. The other option implemented in this system is if the driver is driving the vehicle in the night and if he feel drowsy the system detects it immediately and alarm sound will be initiated to wake up the driver. This system though it won't avoid $100 \%$ accidents at least it will reduce the percentage of accidents. This system is not only to avoid accidents it will also intelligently control the speed of the vehicles and creates awareness amongst the drivers.
\end{abstract}

Keywords: Machine Learning; Traffic Awareness; Driving Awareness; Accidents; Alarm.

\section{Introduction}

In Developing countries like India, it is very difficult to avoid accidents manually instead providing intelligence to the system is always a better solution. [2]. in this paper the solution which is discussed is smart way of providing intelligence to the machine to control the speed of the vehicles whenever or wherever it is essentials. The intelligent system will be embedded inside the vehicle to observe the speed of the vehicle and also to understand the behavior of the driver.

This system works in four step processes.

In first step the speed of the particular road where vehicle is moving is recorded inside the system. This can be done in two ways, the first method where the intelligence system can capture the signboards or speed board's data and record in the system or the information about each and every road is stored in the database which can be fetched by the machine wherever the vehicle is passing.

In the second step, the vehicle speed will be calculated using two machine-learning techniques. In the first method the sensor will be Connected to Speedo meter, which will detect the vehicle speed or image processing techniques, will be implemented to capture the Speedo meter data in certain intervals to calculate the average speed by using machine-learning algorithms.

In third step, the comparison of actual speed in that road and vehicle speed will be done if the driver is driving the vehicle beyond the actual speed same will be intimated to driver by giving beep sound and also red light in the device.
In the fourth step if driver still going beyond the speed after two three indications also the system will send information to nearest police station to take necessary action.

These are the four steps which are discussed above will be made as an Machine Learning Technique and will be embedded inside an Tablet kind of device and which will be embedded in all vehicles[5].

For new cars the manufacturers should embed this device without fail and for the existing car the implementation [6] should take separately in stipulated time in order to successfully implement this product in order to avoid maximum number of accidents which are happening in developing countries like India

\section{Literature survey}

Speed thrills but it also kills! Over Speeding made people to lose not only their life and the people who are on the road and coming opposite side. As per the statistics available with the Traffic police of Bangalore, it shows that out of 1647 road accidents that took place last year, 456 accidents were caused due to over Speeding. It shows more than $25 \%$ accidents are due to over speeding [3].

So, by considering this scenario the government is controlling the speed of the vehicle by fixing the speed limit to 60 for all vehicles But it is not an feasible solution just imagine the scenario where the vehicle is going on express way the where there is no traffic if still we are going in 60 then no use of driving the vehicle on express way by paying additional toll charges.

The other problem is most of the drivers feel sleepy while driving at night. They won't stop and sleeps simply they will be moving 
because of which most of the accidents are taking place in the night. To avoid this Government has taken so many initiatives like by providing free dormitories to drivers etc.., this problem also given solution in this research paper.

\section{Proposed research}

In proposed research two real time problems has been given solution by using Machine Learning Techniques.

1) Controlling the speed of the vehicles

2) Identifying and Stopping the Vehicle when driver is falling asleep.

The device will be manufactured such a way that it has been given intelligence by implementing machine learning algorithms inside this deice to provide the solution for the above two problems.

Type - I: Machine Learning Algorithm to control the speed of the vehicle:

1) Start

2) Read the speed of the current road by reading the sign boards on the road.

3) Read the speed of the vehicle by observing the Speedo meter.

4) Compare the speed of step (2) and step (3).

5) If the speed of step (3) is less than the speed of Step (2) then the device will generate green light and says "Happy Journey" message to driver.

6) Else if the speed of step (3) is greater than the speed of step (2) then the system will produce red light and gives a message to driver to reduce the speed of the vehicle.

7) Step (2) to Step (6) is a continuous process which will be happening in the car at certain intervals in order to control the speed...

8) Stop.

Type - II: Machine Learning Algorithm to control the speed of the vehicle:

1) Start

2) Read the speed of the current road by fetching the information from database, which already stored.

3) Get the information from the sensor which is fixed to Speedo meter to get the speed of the vehicle.

4) Compare the speed of step (2) and step (3).

5) If the speed of step (3) is less than the speed of Step (2) then the device will generate green light and says "Happy Journey" message to driver.

6) Else, if the speed of step (3) is greater than the speed of step (2) then the system will produce red light and gives a message to driver to reduce the speed of the vehicle.

7) Step (2) to Step (6) is a continuous process which will be happenning in the car at certain intervals in order control the speed. 8. Stop.

Machine Learning Algorithm to identify the Stop the Vehicle when driver is falling asleep:

1) Start

2) The system will get information from initially Front Mounted Lane Assistant Camera.

3) In addition, it observes the patterns and informs the driver to take rest.

4) In next step, it will take continuous information from steering angle sensor to identify the driver status.

5) Based on the input from the sensor it will identify whether driver is live or drowsy in order to drive the car.

6) This process will continue in equal intervals to identify the status of the driver.

7) Step (2) to Step (6) will continue whenever there is a disturbance in the either Lane camera or steering sensor.

8) Stop.

\section{Implementation}

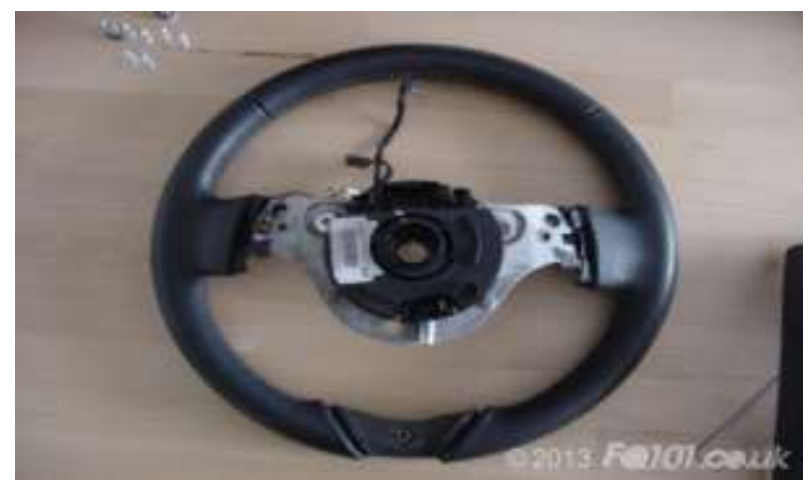

Fig. 1: Steering Wheel with Angle Sensor.

As shown in the Fig.1. The implementation angle senor in the steering wheel will be mainly used to identify the angle of driving carried out by the driver. If the Driver is not following proper angle and there is a disturbance in the angle of direction that information will be sent to Intelligent device fixed and based on the disturbance the device will take the decision either to stop the vehicle or pass the vehicle.[2].

If the driver is not reducing the vehicle speed after certain messages also the intelligent device which is connected will automatically stops the vehicle. [4].

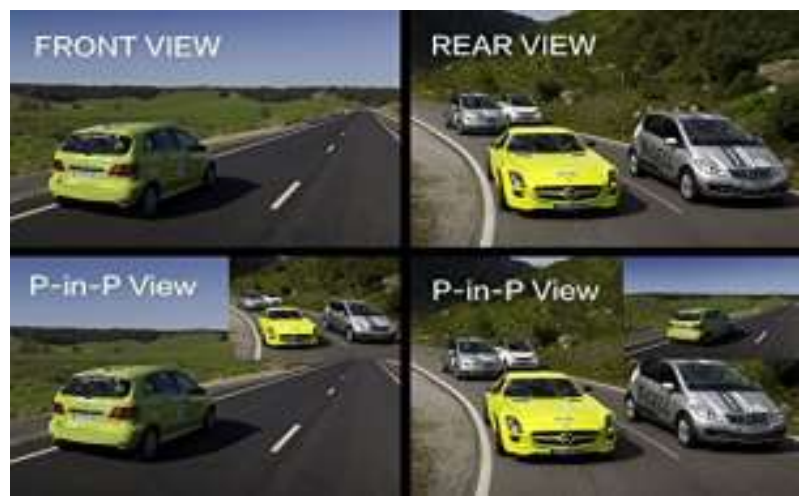

Fig. 2: Lane Camera to Find the Lane Discipline k.

As shown in fig.2. The lane camera will be fixed to car and it will be keep on recording the lane whether driver is going in the correct lane or nor if there is a disturbance in the lane immediately it sends an information to intelligent device in order to control the speed of the vehicle.

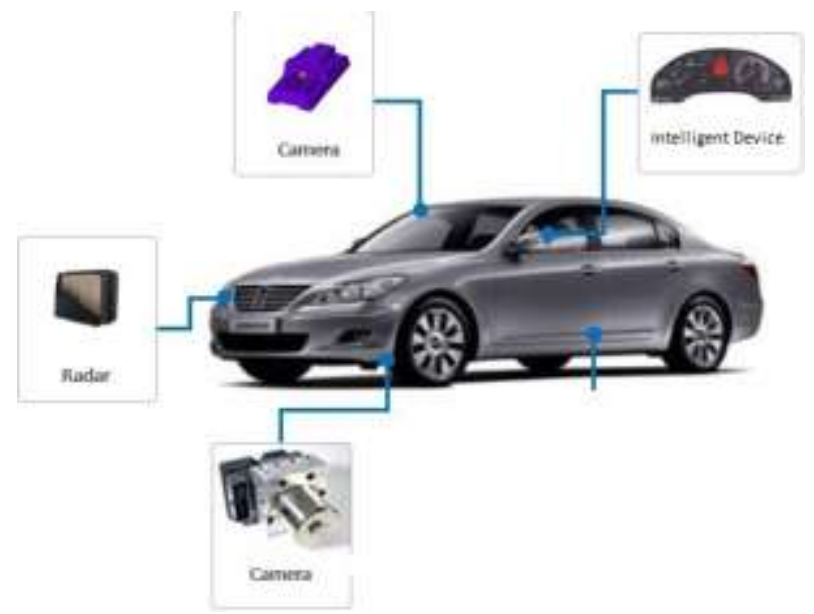

Fig. 3: Car with Intelligent Device and Lane Camera.

As shown in Fig.3. The car manufactures has to take care in order to implement intelligent system to embed inside the car. With which the maximum number of accidents will be avoided. 


\section{Real-time implementation}

This system is a cost-effective solution to control the speed of the vehicle. And this application can be implemented not only in the cars it can also be implemented in any kind of vehicles starting from two wheeler to heavy vehicles like trucks. The main application of this system to implement in School Bus, College Bus, Cabs etc..,[7]

\section{Prototype}

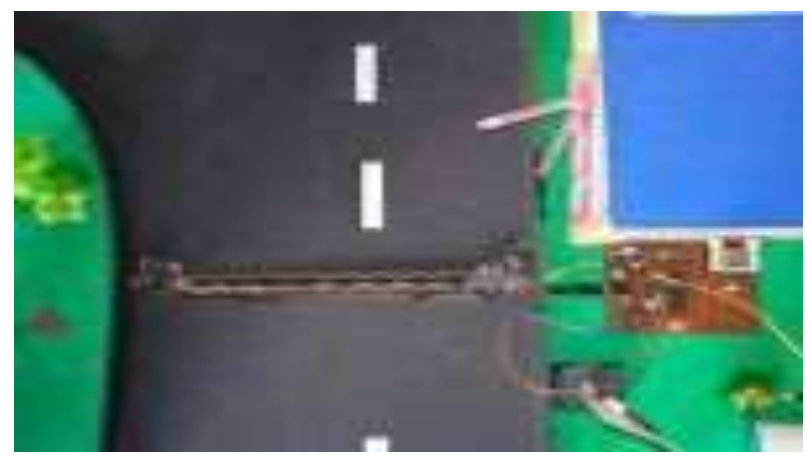

Fig. 4: Prototype of Intelligent System.

The prototype as shown in Fig.4. Is tested in three different scenarios to find the results.

Scenario 1: In the first scenario tested vehicle to read the speed of the road by applying Technique 1 and Technique 2 [1].

Scenario 2: The speed of the vehicle calculated by applying both the techniques.

Scenario 3: The speed of scenario 1 and speed of scenario 2 compared in order to find out either to blink red or green light based on the scenario.

Scenario 4: Lane Camera images are tested in order to know the status of the driver.

Scenario 5: Steering Wheel Sensor is tested in order to know the status of the driver.

By collecting entire data in different scenarios the cumulative decisions are made. [5].

\section{Conclusion}

The Intelligent System is an appropriate solution for the developin countries like India and where there are huge accidents are happening because of the speed and also to avoid the most of the accidents which are happening at nights.

Controlling the vehicle speed is not the dynamic solution, the dynamic solution to avoid accidents I give freedom to drivers to drive and control them intelligently by implementing the intelligent systems like this.

This system alone cannot resolve the entire issue of accident, but it will reduce 30 to $40 \%$ accidents which are happening due to high speed to save the lives of the people.

\section{Future enhancement}

Any Intelligence system success depends not only on the new system built it also depends on the automobile manufacturing companies. In future the system might be improved such a way that a device can be fitted to every vehicle at the time of manufacturing which intern will work as multipurpose device where the message will be sent to the device both as a Display device and it gives voice instruction to the driver. And driver can also listen FM, Songs from the same device.

\section{References}

[1] M S Satyanarayana, Murali Mohan B M, Raghavendra S N "Intelligent Traffic System to Reduce Waiting Time at Traffic Signals for Vehicle Owners", Springer AISC Series, Chapter 31, Volume 668.

[2] Satyanarayana M.S "Reducing the Waiting Time at Toll Gates Using National E-Tolling System "International Research Journal of Advanced Engineering and Science ISSN: 2455-9024.v 1, issue 2, pg. no 100-102. [3].

[3] Rajeshwari sundar, santhoshs hebbar, and varaprasad golla," implementing intelligent traffic control system for congestion control, ambu- lance clearance, and stolen vehicle detection" ieee sensors journal, vol. 15, no. 2, february 2015 .

[4] P. Lakshmi Pallavi, Dr. Mohammed Ali Hussain," Intelligent Traffic Control System using Embedded Web Technology" IRACST - Interna- tional Journal of Computer Science and Information Technology and Se- curity (IJCSITS), ISSN: 2249-9555 Vol. 4, No.2, April 2014.

[5] Khalil m. Yousef, Jamal n. Al-karakil and ali m. Shatnawi” intelligent traffic light flow control system using wireless sensors networks" journal of information science and engineering 26, 753-768 (2010).

[6] Intelligent Traffic Control System" http://en.wikipedia.org/wiki/Intel- ligent Traffic Control System.

[7] https://plus.google.com/+ITCS/videos. 